\title{
Hacia una definición del concepto de colocación: de J. R. Firth a I. A. Mel'čuk
}

\author{
Margarita Alonso Ramos \\ Universidade da Coruña
}

\section{INTRODUCCIÓN}

El objetivo de este trabajo apunta hacia una definición operativa del fenómeno léxico de las colocaciones. Pensamos que una definición precisa de este concepto es imprescindible para poder describir adecuadamente las colocaciones en un diccionario. Señalemos, por lo demás, que las colocaciones han sido un fenómeno léxico desatendido en la lexicografía española por lo que cierta reflexión teórica sobre ellas nos parece necesaria.

El concepto de colocación no ha sido muy utilizado en la bibliografía española. Incluso el término en español no es aceptado por unanimidad ${ }^{1}$. Se suele atribuir a J. R. Firth (1957) la introducción de "colocación"2 como término técnico en la discusión lingüística para referirse a combinaciones habituales de unidades léxicas: dark night, strong argument, heavy smoker.

El término colocación se usa con diferentes sentidos. Unas veces, refiere a combinaciones probables o usuales de dos palabras; otras, a combinaciones restringidas en donde un lexema exige la presencia de otro. Incluso, en ocasiones, se emplea colocación como equivalente a sintagma, como Lyons $^{3}$ cuando habla de colocaciones como el viejo señor.

Antes de poder formular una definición de colocación, creemos que será útil mostrar cómo ha sido entendido el concepto de colocación en diferentes corrientes teóricas. Ahora bien, tal concepto sólo puede ser definido rigurosamente dentro de una teoría específica. Nosotros hemos adoptado el enfoque lexicográfico del Dictionnaire explicatif et combinatoire (DEC) de I. Mel'čuk ${ }^{4}$ (Mel'čuk et al., 1984, 1988, 1992).

1 Vid. R. H. Robins (1971, p. 97), en su traducción española se habla de "posición para referirse al mismo concepto.

2 Según Mel'čuk, el estudioso ruso Vinogradov ya había llamado la atención sobre las colocaciones, anteriormente a Firth. Por otra parte, según T. F. Mitchell (1971, p. 35), el término “colocación" no es original de Firth, quien parece haber sido influido en esta elección por H. E. Palmer.

3 Vid. J. Lyons (1980), p. 227.

4 Este diccionario es elaborado por un equipo de lingüistas de la Universidad de Montreal, bajo la dirección del profesor Mel'čuk. Este modelo ha sido aplicado al ruso y al francés, principalmente. Para una presentación rápida en español de este diccionario y la teoría lingüística en que se basa, vid. M. Alonso Ramos (1989). 
Este marco teórico nos ofrece el dispositivo de las Funciones léxicas, que ha sido principalmente diseñado para describir lexicográficamente las colocaciones.

Organizaremos la exposición por corrientes teóricas y sólo, al final, abordaremos las colocaciones desde la perspectiva del $D E C$, aunque esta perspectiva esté latente a lo largo de todo el trabajo.

Este trabajo se articulará del modo siguiente:

- En el apartado 1, mostraremos las teorías de Firth y Halliday, en donde priman los criterios estadísticos sobre la probabilidad de concurrencia de dos lexemas.

- En el apartado 2, discutiremos el concepto tal y como es entendido por algunos lexicógrafos (Benson, M. Moliner) y semantistas, como Cruse, Hausmann, entre otros.

- En el apartado 3, llamaremos la atención sobre la importancia de la colocación desde la perspectiva de la generación automática de textos.

- En el apartado 4, compararemos los distintos enfoques con el sostenido por el $D E C$. Para ello, explicaremos el concepto de Función léxica y delimitaremos los fenómenos de combinación libre, de frasema o expresión idiomática y de colocación.

\section{DESDE EL CONTEXTUALISMO BRITÁNICO}

Sin adentrarnos en la teoría contextual del significado propuesta por Firth, queremos exponer aquí someramente lo que este autor británico entendía por "colocación".

Para Firth, un aspecto del significado de una palabra es el conjunto de todas las otras palabras con las que se combina. Así, afirma que "uno de los significados de night es su colocabilidad con dark, y de dark, naturalmente, su colocación con night" .

La interpretación del término "significado" en Firth es peculiar. Un enunciado o parte de un enunciado tiene significado sólo si es usado adecuadamente en algún contexto real. Así, oraciones como la de Sapir, The farmer kills the duckling, aunque con "significado gramatical", no tendrán significado a un nivel último de análisis (el semántico) ya que dicha oración no es contextualizable: no puede "ser referida a participantes típicos en algún contexto generalizado de situación"6. Por lo tanto, "significado" equivale a aceptabilidad, a adecuación a un contexto ${ }^{7}$.

Si el término "semántica" se restringe a la contextualización de enunciados en situaciones, hay que preguntarse qué cabe bajo el término "contexto de situación". Ahí entra todo lo que identifica al hablante que vive en una determinada sociedad, con una determinada cultura y con una determinada posición social. Es cierto que la situación

7 Vid. J. LYoNs (1966), p. 290. 
es esencial para una completa descripción lingüística de un enunciado: no hablamos igual en casa que en el trabajo, por poner sólo un ejemplo trivial. Ahora bien, como señala Lyons $^{8}$, hasta que no seamos capaces de sistematizar los contextos de situación, no podremos juzgar la pertinencia de tratar la significación en términos de adecuación situacional.

Los niveles de análisis, entre los que se divide el espectro total del significado, son el fonético y fonológico, el gramatical, el léxico y el semántico. En el componente léxico o colocacional es donde Firth estudia la parte del significado de los lexemas que depende de su tendencia a coocurrir. Ahí se tendrá que determinar qué colocaciones son aceptables o inaceptables pero no utiliza criterios claros. Firth, en el artículo donde introdujo la noción de colocación, estudió la frecuencia de colocaciones particulares para caracterizar el estilo de un autor. Distinguía entre colocaciones usuales e inusuales. Estas últimas serán las importantes para los estudios estilísticos.

Lyons ${ }^{9}$ compara este tratamiento del significado léxico con la teoría distribucional del significado, en la línea de Harris: dos lexemas tienen el mismo significado si tienen la misma distribución. Si se llega a probar que la misma distribución de ciertos lexemas se correlaciona con un mismo significado, todavía habrá muchas relaciones de significado que no pueden ser derivadas de criterios distribucionales. Hoy, la teoría distribucional del significado puede ser rechazada. Lyons es categórico en el rechazo de las colocaciones como parte del significado de una palabra:

\begin{abstract}
"Llegamos a aprender el significado de muchas palabras en virtud de oírlas o verlas en varios contextos verbales. Pero nuestra capacidad de inferir su significado de los contextos en que son empleadas, verbales o situacionales, depende de nuestra inteligencia o 'sentiment linguistique', de nuestro conocimiento del significado de otras palabras en esos contextos y de nuestra comprensión de las oposiciones paradigmáticas y equivalencias que se sostienen entre la palabra en cuestión y otras palabras que podrían haber aparecido, pero de hecho no aparecen, en el mismo punto en el texto" 10 .
\end{abstract}

Nosotros coincidimos con Lyons en su crítica a Firth con respecto a la identificación del significado de una palabra con el conjunto de sus colocaciones. Ahora bien, es cierto que en muchas ocasiones, el hecho de que dos lexemas concurran está determinado por su significado. Nosotros no diríamos que el significado de rubio es su colocabilidad con pelo pero sí que el hecho de que estos dos lexemas concurran está determinado por el significado de rubio: en su definición, debe haber una mención a 'pelo'.

Lyons ${ }^{11}$ es más abierto al considerar que la potencialidad de colocación puede ser parte del significado de un lexema pero advierte que "no debemos pasar desde el extremo de decir que las colocaciones de un lexema están determinadas por su significa-

8 Vid. J. LyONS (1966), p. 292.

9 Vid. J. LYONS (1966), p. 295.

10 Vid. J. LYONS (1966), p. 296.

11 Vid. J. Lyons (1966), p. 249. 
do (...) al extremo opuesto de definir el significado de un lexema como si no se tratara más que del conjunto de sus colocaciones".

Pensamos que el mérito de Firth está en haber despertado el interés por la coocurrencia léxica pero el nivel colocacional, que propuso, basado en la frecuencia de aparición, resulta de un interés limitado.

Entre los lingüistas neo-firthianos ${ }^{12}$, destaca Halliday. En su artículo de 1966, propone un nivel lingüístico, llamado "lexis", independiente de la gramática, para el estudio de las colocaciones.

Halliday (1966) defiende el establecimiento de una teoría léxica independiente de la teoría gramatical. El lexis es a la semántica léxica como la gramática es a la semántica gramatical. Se trata, pues, de estudiar el léxico como forma léxica, de diferente tipo que la forma gramatical. Introduce la noción de "lexicalidad", paralela a la de "gramaticalidad".

Este autor plantea la cuestión de si las relaciones léxicas pueden ser mejor tratadas dentro o fuera de la gramática. Para ello, ofrece el ejemplo de dos adjetivos strong y powerful, que pueden ser yuxtapuestos a argument. Pero strong no está siempre en la misma relación con powerful: strong pero no powerful puede combinarse con tea, y powerful, pero no strong, puede combinarse con car. Así, a strong car o powerful tea serían tratados como "aléxicos" (no como "agramaticales"). La relación paradigmática de estos adjetivos depende de la relación sintagmática en la que cada uno entra.

Para Halliday, las relaciones entre estos lexemas no son tratadas adecuadamente por la gramática. Ambos adjetivos tendrían que ser asignados a la misma clase, y así, esperaríamos encontrar a strong car, de igual modo que tenemos a powerful car. Las mismas relaciones de coocurrencia se encuentran también en otras unidades: strong, strongly, strength forman colocación ("collocate") con argue, argument y con tea; y por otro lado, power, powerful, powerfully forman colocación con argue y argument y con car. La gramática consideraría strong, strength como elementos diferentes y serían asignados a diferentes clases. Sin embargo, para estas relaciones colocacionales, las diferencias de clases gramaticales son irrelevantes. Halliday trata los elementos de la misma familia léxica como una sola unidad léxica.

Las relaciones léxicas pueden ser puestas en paralelo con las relaciones gramaticales. Al nivel sintagmático, la categoría teórica gramatical de "estructura" es comparable a la categoría léxica de "colocación". Al nivel paradigmático, en lugar de "sistema", el lexis requiere la categoría de "conjunto" (“set”): el conjunto estará formado por todos los elementos léxicos con la misma coocurrencia. Así, strong y powerful pertenecerán al mismo conjunto en relación con argue pero a diferentes conjuntos en relación con otros lexemas.

Con la división entre un nivel léxico y un nivel gramatical, Halliday no pretende decir que haya estructuras léxicas distintas de las gramaticales. Según él, todas las formas lingüísticas entran en ambas estructuras. Serán tratadas como gramaticales cuan-

12 Vid. también T. F. Mitchell (1971), quien se aparta de los neo-firthianos y que considera que las colocaciones deben ser estudiadas dentro de la gramática). 
do son descritas gramaticalmente y como léxicas cuando son descritas léxicamente. Con todo, señala que ciertos elementos pueden ser mejor explicados desde un punto de vista gramatical o léxico. Así, por ejemplo, los elementos a y of son muy poco restringidos colocacionalmente y son perfectamente descritos en la gramática. En contraste, strong, desde un punto de vista gramatical, sólo se puede decir de él que es miembro de la clase de los adjetivos pero, colocacionalmente, es muy restringido. Se puede establecer, por tanto, una escala de "más gramatical" a "más léxico": un elemento será más gramatical si es óptimamente descrito por la gramática.

En el análisis léxico, se tienen en cuenta solamente las restricciones de coocurrencia. Para establecer el entorno colocacional de una unidad léxica, se observa la frecuencia de esa unidad en un entorno dado en relación con su frecuencia total de aparición.

El criterio para definir un "conjunto" léxico es, entonces, la potencialidad de aparición. Dos apariciones de un elemento asignadas a diferentes conjuntos pueden ser tratadas como diferentes unidades léxicas. Y a la inversa, strong, strength, strongly serán tratados como la misma unidad léxica, ya que pertenecen al mismo conjunto. Las unidades léxicas que muestren un cierto grado de probabilidad en su comportamiento "colocacional" son asignados al mismo conjunto. Por ejemplo, bright, shine, light son agrupadas en el mismo conjunto ya que muestran una similar potencialidad de aparición en el entorno de sun y en el de moon. La aparición de una unidad en un entorno colocacional sólo puede ser tratada en términos de probabilidad.

Las colocaciones serán juzgadas según el grado de "lexicalidad". Ante una colocación dada, un hablante nativo no la acepta o la rechaza sino que la tratará como más o menos aceptable. Aunque Halliday se refiere a una investigación sobre textos, sugiere que quizá las colocaciones de una unidad léxica dada podrían ser facilitadas por una encuesta en la que se le pida a un sujeto listar veinte unidades léxicas que esperaría encontrar en colocación con esa unidad.

El empeño de Halliday en establecer un nivel léxico, en donde se introduzcan criterios formales, se debe a su concepción firthiana de la semántica. Las relaciones semánticas o "contextuales" son externas: entre lo que es lengua y "no lengua". Las relaciones formales son internas, dentro de la lengua. Así, para él, si se iguala el "lexis" con la semántica, no se podrá tratar el léxico de una manera formal.

Es difícil valorar el "lexis" de Halliday sin adentranos a fondo en su teoría lingüística. Aunque nosotros apoyamos un estudio formal del léxico, no por ello defendemos una delimitación tajante entre el estudio léxico y el estudio gramatical ya que, a menudo, varios aspectos se interrelacionan. Por otra parte, ceñir el estudio léxico a las relaciones colocacionales no nos parece apropiado.

Proponer el "conjunto" y la colocación como nuevas categorías teóricas para tratar el léxico no nos parece que ayuden demasiado a estudiar formalmente el léxico. Decir que strong y powerful forman un conjunto en relación con argument pero no en relación con car no sirve de mucho si esa información no es operativa; es decir, ¿dónde se necesita la información de que strong y powerful coocurren con argument? Creemos que únicamente en la entrada lexicográfica de argument. Si resulta ser el caso que strong y powerful forman colocación con otros lexemas además de con argument, entonces sí sería de alguna validez teórica intentar encontrar alguna generaliza- 
ción que explicara estas colocaciones. Los "conjuntos léxicos" no tienen sentido tratados como constructos teóricos, si no tienen cierto grado de generalidad.

La noción de colocación, tal y como es tratada por Halliday, no nos parece tampoco interesante. Las colocaciones son definidas en términos de probabilidades y frecuencias. Si después del análisis de grandes cantidades de textos, se encuentra que gaviota coocurre frecuentemente con blanca, no por ello debería concluirse que gaviota blanca es una colocación.

Hemos elegido ese ejemplo precisamente porque es el que emplea Coseriu (1981) para distinguir sus solidaridades léxicas de los "virtuemas" (combinaciones usuales y frecuentes) de Pottier. Coseriu es tajante a este respecto:

"La frecuencia de la combinación del adjetivo blanche con el sustantivo mouette depende de nuestro conocimiento de las gaviotas, no de la lengua francesa, ya que 'blanc, blanche' no implica el rasgo distintivo 'para las gaviotas': es por tanto, un hecho de gaviotas, no un hecho de lengua" 13 .

En otro lugar, Coseriu ${ }^{14}$ afirma claramente que "la probabilidad estadística general de las combinaciones no tiene prácticamente nada que ver con las solidaridades y no es prueba de su existencia".

Coincidimos plenamente con la postura de Coseriu. El hecho de que dos lexemas coocurran frecuentemente no es prueba de que exista una colocación. La coocurrencia de los lexemas puede estar determinada por su significado y esto es independiente de que ambos lexemas aparezcan frecuentemente en los textos.

En una obra posterior, Halliday y Hasan ${ }^{15}$, estudiando la cohesión léxica, recurren al concepto de colocación para referirse a elementos que tienden a compartir el mismo entorno léxico. Entre los ejemplos de colocaciones que ahí ofrecen, encontramos pares de lexemas, vinculados por relaciones paradigmáticas, que pueden coocurrir si bien no en el mismo sintagma. Por ejemplo: boy...child, boy...girl, car...brake. Otras relaciones son evocadas, sin intentar clasificarlas semánticamente, como laugh...joke, garden...dig, ill...doctor, try...succeed.

Halliday no es el único en emplear el término "colocación" para referirse a palabras que coocurren frecuentemente. También Evens ${ }^{16}$, identificando relaciones sintagmáticas con las colocaciones, señala que swim está relacionado sintagmáticamente con water, pool y bathing cap. Para nosotros, el hecho de que estos lexemas coocurran frecuentemente no es una razón para considerarlas como colocaciones. Si coocurren es una consecuencia de que los textos no pasan de un tema a otro sino que siguen un hilo argumental.

13 Vid. E. COSERIU (1981), p. 184.

14 Vid. E. Coseriu (1981), p. 160.

15 Vid. Halliday y Hasan (1976), p. 295.

16 Vid. Evens (1988), p. 7. 
DESDE LA SEMÁNTICA LÉXICA Y LA LEXICOGRAFía

En el concepto de colocación, tal y como hasta ahora ha sido mostrado, entran combinaciones frecuentes de lexemas, ya sea apareciendo juntos o, simplemente, coocurriendo en la misma secuencia discursiva. Con todo, en lexicografía y en semántica léxica, es más frecuente el uso del término "colocación" para referirse a lexemas que aparecen formando un sintagma.

El criterio de la frecuencia o de la recurrencia para determinar lo que es una colocación viene desde lejos en la bibliografía lingüística, aunque sin estar apoyada en criterios estadísticos. Bally (1951) advirtió un tipo especial de combinaciones de palabras, al que llamó "groupement usuel":

“hay serie o agrupación usual cuando los elementos del grupo conservan su autonomía, aunque dejando ver una afinidad evidente que los aproxima, de tal manera que el conjunto presenta contornos delimitados y da la impresión de déjá vu".

Entre sus ejemplos destacan los ya célebres gravement malade y grièvement blessé, en donde el intercambio de adverbios es imposible. Bally percibió la existencia de colocaciones pero se centró en el carácter "usual" de la combinación.

Siguiendo a Hausmann ${ }^{17}$, podemos agrupar la combinación probable de Halliday con la combinación usual de Bally para mostrar que ambas concepciones de la colocación son rechazables. La cantidad de combinaciones probables o usuales es enorme. Una combinación como mirar un árbol será probable e incluso frecuente pero no por ello esta combinación es una colocación. Está formada según las reglas de la sintaxis, es completamente libre: el verbo mirar se puede combinar con todo lo que puede ser mirado. Y esta libertad abarca a todas las lenguas en donde exista un verbo equivalente a mirar.

Sin embargo, hay otras combinaciones restringidas en donde dado un lexema como base, no podemos combinarlo con cualquier otro para expresar un sentido dado. Por ejemplo, en un esfuerzo ímprobo, una actividad febril o una lucha encarnizada, no podemos intercambiar los adjetivos a pesar de que todos significan en esas combinaciones 'intenso'. Es cierto que estas combinaciones son frecuentes. Nombre y adjetivo aparecen juntos frecuentemente pero no puede ser de otro modo ya que la elección del adjetivo es obligatoria. Igualmente frecuente es la combinación comida pantagruélica, no porque esta expresión aparezca a menudo en los textos sino porque si queremos predicar de una comida el sentido 'excesivamente abundante', tendremos a nuestra disposición el lexema pantagruélica. En muchas ocasiones, la restricción léxica es evidente cuando intentamos traducir literalmente este tipo combinaciones a otras lenguas. Así, mientras en español, tenemos dar un paseo, en francés, es imposible *donner une promenade; el verbo escogido es faire.

Obsérvese que no se trata de expresiones idiomáticas o locuciones, en sentido estricto. Como veremos en el último apartado, en las locuciones, el sentido total de la ex- 
presión no refleja el sentido de sus partes constituyentes. Sin embargo, en las colocaciones, cada constituyente aporta una parte de sentido a la expresión en conjunto.

Algunos semantistas y lexicógrafos han percibido la posición intermedia de la colocación entre la expresión idiomática y la combinación libre pero siguen centrándose en su carácter habitual, usual o frecuente. Así, por ejemplo, Cruse ${ }^{18}$ utiliza el término "colocación" para referirse a "secuencias de unidades léxicas que coocurren habitualmente pero que, no obstante, son completamente transparentes en el sentido de que cada constituyente léxico es también un constituyente semántico". Entre sus ejemplos están fine weather, torrential rain, light drizzle, heavy drinker.

Más adelante, Cruse ${ }^{19}$ habla de "restricciones colocacionales" para tratar la "impropiedad" ("inappropriateness") de una combinación. La impropiedad será subsanada reemplazando un elemento de la combinación por su sinónimo cognitivo. Así, en la siguiente oración

\section{1) Have you grilled the bread?}

si sustituimos grilled por toasted, la impropiedad desaparece. Cruse ${ }^{20}$ define las restricciones de colocación como "restricciones de coocurrencia que son irrelevantes a las condiciones veritativas". Si el interlocutor al que se dirige el que haya formulado la anterior pregunta ha tostado realmente el pan, su respuesta cooperativa sería "Sí, aunque él no haya "grilled" el pan sino que lo ha "toasted".

Cruse $^{21}$ distingue entre restricciones colocacionales "sistemáticas”, “semi-sistemáticas" e "idiosincrásicas”. En las primeras, se puede explicar semánticamente la restricción. Por ejemplo, la diferencia entre grill y toast estriba en que el primer verbo se utiliza para cocinar algo crudo mientras que el segundo para cosas ya cocinadas. En las restricciones semi-sistemáticas, hay también ciertas propiedades semánticas pero con excepciones. Las idiosincrásicas son casos de restricciones completamente arbitrarias. Los ejemplos que ofrece son una serie de sinónimos cognitivos que se combinan con distintos nombres, al azar: flawless argument, immaculate kitchen, impeccable taste. Estos adjetivos también en español están restringidos colocacionalmente: una cocina puede estar inmaculada o impecable pero el gusto no puede ser inmaculado; intachable se combina más con nombres que designen conducta o comportamiento.

A nuestro modo de ver, las restricciones colocacionales idiosincrásicas son el punto débil de la argumentación de Cruse. La impropiedad de una combinación léxica no puede ser subsanada siempre por un sinónimo cognitivo. Suponiendo que inmaculado, intachable e impecable sean sinónimos cognitivos, la impropiedad de * una conducta inmaculada sólo es subsanada si sustituimos el adjetivo por intachable; la combinación con impecable parece menos natural.

Las colocaciones se caracterizan por tener una direccionalidad: uno de los lexemas selecciona otro. En estos ejemplos, es el nombre el que selecciona un determina-

18 Vid. D. A. CRUSE (1986), p. 40.

19 Vid. D. A. CRUSE (1986), p. 107.

20 Vid. D. A. CRUSE (1986), p. 279.

21 Vid. D. A. CRUSE (1986), p. 281. 
do adjetivo. Tenemos el lexema conducta y queremos expresar que es 'buena y no puede ser criticada'. La selección del lexema intachable viene dada por el lexema conducta ya que si se parte de otro lexema como honor, el mismo sentido sería expresado por inmaculado.

Cruse, sin embargo, trata la direccionalidad en sentido inverso. En las construcciones "núcleo-modificador" trata como "seleccionador" al modificador y piensa que la impropiedad tiene lugar cuando las presuposiciones del seleccionador no son satisfechas por el seleccionado. Es cierto que embarazada presupone el componente semántico 'hembra' en el núcleo de una construcción. Sin embargo, ¿qué componente puede presuponer impecable al nombre que modifique? Creemos que Cruse no ha discernido adecuadamente las restricciones de selección de las de colocación. En el caso de a pregnant student, la coocurrencia es libre, está determinada semánticamente por los sentidos de los lexemas. Sin embargo, en el caso de impeccable taste, la coocurrencia es restringida: es el nombre el que elige un modificador determinado y no otro. Cuando se trata de una colocación, el seleccionador es siempre el nombre, tanto en las construcciones nombre-adjetivo como en verbo-nombre.

En nuestra opinión, Hausmann ${ }^{22}$ ha tratado certeramente la direccionalidad de las colocaciones. Este autor define la colocación como una "combinación restringida y orientada". En una colocación como célibataire endurci, el nombre es la "base" y el adjetivo, el "colocativo" ("collocatif"). La base es autónoma, no necesita del colocativo para ser definida. En cambio, la definición del colocativo necesita ser completada por la base. Hausmann ${ }^{23}$ piensa que esa es la razón por la que diccionarios como el Petit Robert incluya más frecuentemente la colocación en el artículo del colocativo. Es cierto que es difícil definir craso, por ejemplo, sin mencionar 'error'. Ahora bien, como el propio Hausmann indica, los diccionarios de definiciones (frente a los de colocaciones) ayudan poco al que escribe un texto: el usuario, normalmente, conoce la base y busca el colocativo que se acomode a un sentido dado.

Entre los diccionarios españoles, sin duda, el de M. Moliner es el que más colocaciones proporciona y lo hace muy a menudo en la entrada de la base. Por ejemplo, en la entrada de sueño, se indica, entre paréntesis y antes de la definición, la serie siguiente de colocativos:

“acometer, apoderarse, entrar, invadir; rondar; coger, conciliar, entregarse al; espantar"

En ocasiones, la dimensión sintagmática del colocativo es explicitada también en su definición. Así, ahí mismo, encontramos S.v. rondar 5:

“Estar cierta cosa, como el sueño o una enfermedad, a punto de apoderarse de alguien".

23 Vid. F. Hausmann (1979), p. 192. 
Con todo, M. Moliner no fue sistemática. Así, la colocación cariño entrañable es descrita en la entrada del colocativo. Encontramos s.v. entrañable:

“(aplicado a cariño o nombre equivalente). Verdadero y profundo".

A pesar de algunas inconsistencias y so pena de ser acusada de tener devoción por María Moliner, tenemos que decir que su diccionario es el que mejor trata las colocaciones en español. Sin embargo, a lingüistas como Gregorio Salvador ${ }^{24}$, les parece que expresiones como sueño ligero, profundo, cariño entrañable, ruido infernal, entre otras, son "frases hechas, que desde luego no pintan nada en un diccionario". Disentimos completamente de esta opinión. En primer lugar, no se trata de "frases hechas" ya que cada uno de los lexemas contribuye semánticamente al sentido global. En segundo lugar, el hecho de que estas expresiones no sean solidaridades léxicas no es razón para desecharlas del diccionario. En tercer lugar, defendemos la presencia de las colocaciones en el diccionario. Un hablante no nativo puede aprender el significado de una base por aislado, mientras que, como señala Hausmann 25 "el aprendizaje de los colocativos sólo es concebible en colocación".

Ya que hemos mencionado al hablante no nativo, debemos decir que los diccionarios de colocaciones le resultan imprescindibles si quiere dominar una lengua segunda. Por lo que sabemos, no existe ningún diccionario de ese tipo en español. En inglés, desde 1986, contamos con un diccionario de colocaciones, especialmente destinado a aprendices de inglés. Se trata del BBI Combinatory Dictionary of English: A Guide to Word Combination ${ }^{26}$, de M. Benson, E. Benson y R. Ilson (en adelante, BBI). Veamos cuál es la concepción de la colocación en este diccionario.

En la introducción (p. IX), los autores se refieren a las colocaciones como "sintagmas y construcciones fijas, identificables, no idiomáticas". Esta descripción tiene el mérito de situar las colocaciones en una posición intermedia entre las combinaciones libres y las expresiones idiomáticas ${ }^{27}$. Presentan cierto grado de fijación dado que se las puede reconocer pero no están completamente petrificadas. En otra obra de los mismos autores ${ }^{28}$ señalan que las colocaciones son "psychologically salients".

En el $B B I$, utilizan como sinónimo de colocación, “combinación recurrente". De nuevo, nos encontramos pues con el criterio de la frecuencia para considerar una combinación dada como colocación. Según este criterio, el $B B I$ "incluye los verbos bake, boil, chop,... (como en bake me a cake)...; no incluye verbos menos frecuentemente usados en las mismas construcciones (barbecue, braise, brown,...)" (p. XV). No entendemos por qué bake a cake es tratada como colocación mientras barbecue a hamburger no lo es. El criterio de frecuencia puede ser válido como criterio de elección de la nomenclatura del diccionario pero no como definición de colocación. La utilidad de in-

24 Vid. G. Salvador (1989-1990), p. 363.

25 Vid. F. Hausmann (1979), p. 192.

26 Para una visión global de este diccionario, vid. BEJoINT y THOIRoN (1987).

27 Con todo, podemos encontrar en el $B B I$ algunos frasemas como el célebre to kick the bucket bajo la entrada del nombre.

28 Vid. M. BENSON et al. (1986b), p. 252. 
cluir sólo combinaciones frecuentes es muy discutible desde el punto de vista del usuario. De hecho, el aprendiz puede tener más problemas con una combinación poco frecuente, precisamente por su rareza. Por ejemplo, to barbecue a hamburger for me / ?to barbecue me a hamburger.

El $B B I$ proporciona dos tipos de colocaciones, que los autores llaman "colocaciones gramaticales" y "colocaciones léxicas". Las primeras se componen de "una palabra dominante", nombre, adjetivo o adverbio, seguida de una preposición o de una estructura sintáctica (oración de infinitivo, oración introducida por that). Así, por ejemplo, en la entrada de pleasure se indica que rige una oración de infinitivo: a pleasure to do it.

En las colocaciones léxicas sólo intervienen nombres, adjetivos, verbos y adverbios. En la entrada de regards, se proporciona la siguiente serie de adjetivos: best, cordial, friendly, kind, sincere, warm, warmest. En la entrada de murder, encontraremos el verbo to commit. Para mostrar la diferencia entre las colocaciones léxicas y las "combinaciones léxicas libres", los autores recurren de nuevo al criterio de frecuencia: "las combinaciones léxicas libres son aquellas en que los dos elementos no coocurren repetidamente" (p. XXIV). Como ejemplo, ofrecen una construcción como condemn murder. Dudamos que esta construcción sea menos frecuente que commit murder. Quizás sí, pero lo que las distingue es que en la combinación libre no operan otras reglas que las de la sintaxis inglesa, mientras que en la colocación, el verbo es seleccionado por unos pocos nombres que signifiquen 'crimen', como los propios autores señalan.

Los autores distinguen varios tipos de colocaciones gramaticales y léxicas, que describen en su introducción. En las entradas, las colocaciones léxicas preceden a las gramaticales. Ahora bien, si las gramaticales aparecen siempre, las léxicas pueden fallar. No hemos encontrado ninguna explicación para esto. Así, en las entradas de los verbos grill y toast solamente encontramos información gramatical: la alternancia del dativo que se da en, por ejemplo, he grilled a hamburger for us / he grilled us a hamburger. Si ahora recordamos el ejemplo que proponía Cruse (1986) como casos de restricciones colocacionales, * to grill a bread, el $B B I$ no nos informa de esta restricción. No es fácil decidir si la restricción propuesta por Cruse es realmente colocacional o más bien se trata de coocurrencia libre, determinada por el significado de to grill y de to toast.

Los tipos de colocaciones léxicas ${ }^{29}$ son los siguientes:

1. Verbo que significa 'creación' o 'activación' y nombre/pronombre. Ejs.: inflict a wound, launch a missile.

2. Verbo que significa 'erradicación' y nombre. Ejs.: revoke a license, rescind a tax.

3. Adjetivo y nombre: strong tea, warmest regards, a chronic alcoholic.

4. Nombre y verbo que significa "una acción característica de la persona o cosa designada por el nombre”. Ejs.: adjectives modify, blood circulates.

5. Nombre y nombre, en donde uno designa la unidad y el otro el conjunto. Ejs.: a colony of bees, a bouquet of flowers, a bit of advice, an article of clothing. 
6. Adverbio y adjetivo. Ejs.: deeply absorbed, strictly accurate, sound asleep.

7. Verbo y adverbio. Ejs.: affect deeply, anchor firmly, appreciate sincerely.

Las colocaciones gramaticales no las trataremos aquí ya que corresponden al régimen. A nuestro modo de ver, decir que un lexema verbal dado rige una oración de infinitivo o que admite la alternancia de dativo no es información colocacional. Por supuesto que esa información debe aparecer en una descripción del léxico ${ }^{30}$ pero no vemos el interés de tratarla como colocación. De hecho, ni siquiera se conforma a la definición de colocación dada en el BBI. El adjetivo angry junto con la preposición at o with no forma una combinación fija, identificable, no idiomática y recurrente. Creemos que la coocurrencia sintáctica y la coocurrencia léxica deben ser tratadas separadamente.

Un diccionario de colocaciones permite incluir los colocativos en la entrada de la base. Así, en la entrada de murder, aparecerá el verbo to commit. Esto no quiere decir que el verbo no dispondrá también de una entrada. En ella, se incluirá información gramatical y, si es el caso, los colocativos que se combinen con la base verbal. Con todo, la direccionalidad no es siempre respetada en el $B B I$. Encontramos la colocación to browse through a book en la entrada del verbo. Como señalan Bejoint y Thoiron ${ }^{31}$, "esto es particularmente molesto si se intentan imaginar las condiciones en las que el usuario necesitará la información".

Además de la elección de lemas, un diccionario de colocaciones tiene que decidir qué colocativos incluye en una entrada dada. Los autores del $B B I$ excluyen, por ejemplo, expresiones como to cause damage argumentando que las combinaciones con el verbo to cause, a pesar de que expresan el mismo significado de 'creación' o 'activación' (colocaciones del tipo 1), "forman un número casi ilimitado de combinaciones" (p. XXV). Ahora bien, el problema es que si no incluimos esa combinación, el usuario puede pensar que la única manera de expresar 'causar daño' es to inflict damage. Hausmann alude a este mismo problema a propósito de avoir des doutes:

"la ausencia de avoir al lado de concevoir no podría ser interpretada más que como un indicio de la inaceptabilidad de la combinación avoir des doutes"32.

Creemos que para evitar esto, hay que explicitar desde el principio si la lista de colocaciones está cerrada o es sólo una muestra.

Desafortunadamente, en el $B B I$ no utilizan criterios semánticos. Así, en una misma entrada pueden aparecer sentidos distintos del lema. Por ejemplo, en la entrada de degree, aparecen los colocativos correspondientes al sentido 'título académico' y al sentido 'nivel' e incluso la forma adverbial to a high degree. Asimismo, algunas de las colocaciones aparecen glosadas y otras no, sin que sepamos cuál es el criterio de su elección.

Aunque el $B B I$ peque, en algunos aspectos, de poco rigor y de falta de criterios semánticos, creemos que su labor es encomiable. Cumple un papel importante en la lexicografía inglesa, que puede ser envidiado por la lexicografía española.

32 Vid. F. Hausmann (1979), p. 193. 
DESDE LA GENERACIÓN AUTOMÁtICA DE LENGUA NATURAL

Si en la teoría lingüística las colocaciones han sido escasamente estudiadas y mal entendidas, los lingüistas que trabajan en la generación automática de lengua natural han captado adecuadamente la naturaleza especial de este fenómeno. No resulta extraño si pensamos que la perspectiva de estos trabajos es la producción, la codificación de textos. En la generación automática de lengua natural, hay dos fases, que podemos simplificar en el "qué decir" y en el "cómo decirlo". En esta segunda fase, hay que elegir el lexema que exprese un sentido dado, pero esta elección puede estar condicionada. Para generar, por ejemplo, la expresión actividad febril, el sistema debe saber que dado el lexema actividad, si queremos predicar que es 'muy intensa', la elección del lexema que exprese este sentido no es libre: *actividad viva. Sin embargo, no hay ningún problema en analizar la expresión actividad febril. El sistema ("parser") la analizará como un nombre y un adjetivo sin requerir ningún conocimiento especial.

Cumming (1986) llamó la atención sobre estas combinaciones, especialmente interesantes para la generación automática. Distingue certeramente las colocaciones de las expresiones idiomáticas ("idioms"). Las primeras son "composicionales y pueden ser semánticamente transparentes al oyente pero requieren conocimiento específico por parte del hablante para producirlas correctamente" (p. 18). Sin embargo, las expresiones idiomáticas, del tipo to kick the bucket, son "no composicionales, no predecibles del significado de sus partes" (p. 16).

Después de examinar varios sistemas de procesamiento de lengua natural, Cumming ${ }^{33}$ constata que ninguno trata explícitamente las colocaciones. Unos las confunden con las restricciones de selección y otros con las expresiones idiomáticas. Esta autora critica esta confusión ya que, por una parte, se pierde la distinción entre propiedades semánticas generales y propiedades léxicas idiosincrásicas. Por otra, al confundirlas con las expresiones idiomáticas, no se tiene en cuenta que las colocaciones son composicionales semánticamente.

S. Nirenburg e I. Nirenburg ${ }^{34}$ también constatan que las restricciones de coocurrencia no pueden ser tratadas solamente en términos de restricciones de selección. Señalan que, por ejemplo, el verbo to shed se combina con tears y no podemos decir * shed water out of a bucket. Estas relaciones colocacionales no son definidas sobre representaciones de significado sino sobre unidades léxicas. Para que un sistema automático pueda producir colocaciones correctamente debe disponer de un léxico en donde se encuentre esta información.

Heid y Raab (1989) proponen estructurar el conocimiento colocacional en el léxico de un sistema de generación multilingüe. Conocen el tratamiento lexicográfico de las colocaciones y siguen a Hausmann y a Mel'čuk. Inciden también sobre la idea de que las restricciones de selección no son adecuadas para la elección de los colocativos en el proceso de lexicalización. Como criterio heurístico, sugieren que la base debe ser lexi-

34 Vid. S. NiREnburg e I. Nirenburg (1988), p. 471. 
calizada antes que el colocativo ya que éste depende del lexema que se escoja como base. Proponen utilizar las FFLL como medio de formalizar la información colocacional.

En un trabajo posterior, Heid ${ }^{35}$ perfila más detalladamente su concepto de colocación. Distingue tres niveles de análisis:

- semántico: la base puede ser descrita autónomamente mientras que el colocativo no;

- léxico: la elección del lexema semánticamente no autónomo está restringida por las propiedades léxicas del lexema autónomo;

- sintáctico: las colocaciones son formuladas según ciertas estructuras de categorías, en donde el primer elemento es la base: nombre + nombre, nombre + verbo, verbo + adverbio, etc.

\section{Desde el DICTIONNAIRE EXPLICATIF ET COMBINATOIRE (DEC)}

Si bien no podemos caracterizar aquí en profundidad el modelo lexicográfico representado por el $D E C$, unas pequeñas indicaciones son necesarias para mostrar desde esta perspectiva el concepto de colocación. Las principales características del $D E C$ son las siguientes:

- El DEC está ligado íntimamente a una teoría lingüística: la Teoría Sentido-Texto, propuesta por Mel'čuk y Zholkovsky (vid. por ejemplo Mel'čuk y Zholkovsky 1970).

- No es un diccionario comercial. Está concebido como un léxico teórico.

- Está orientado más hacia la codificación que hacia la descodificación.

- Cada artículo lexicográfico del DEC comprende tres secciones: semántica (definición del lema), sintáctica (régimen sintáctico) y léxico-combinatoria.

Esta última sección es la que nos interesa aquí. Está destinada a dar cuenta de la coocurrencia léxica restringida del lexema, lema del artículo. Podemos hablar de coocurrencia léxica restringida cuando para expresar un sentido ' $C$ ', con el lexema $A$ ya escogido, la elección de B está determinada léxicamente por A. Así, por ejemplo, para expresar el sentido 'intenso' con el lexema odio, la elección de mortal está determinada por el lexema odio. La elección de mortal no sería válida si en lugar de tener el lexema odio, tuviéramos el lexema ira (cf. ira ciega vs. *ira mortal). Los lexemas A (odio) y B (mortal) forman entonces una colocación, al igual que ira y ciega. Para describir la coocurrencia léxica restringida, los autores del $D E C$ utilizan el aparato de las Funciones léxicas (FFLL).

Grosso modo, una FL es un sentido o un papel semántico-sintáctico tal que su expresión depende del lexema al que esta FL se aplica. Se trata de combinaciones como 
odio MORTAL [Magn(odio)], ira CIEGA [Magn(ira)], prohibir TERMINANTEMENTE

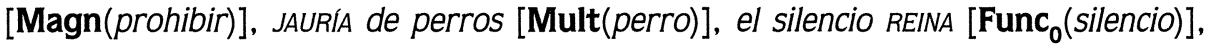
DAR un beso [0 per $_{1}$ (beso)], hacer una caricia [0 per $_{1}$ (caricia)], etc. El lexema al cual la FL se aplica es llamado "palabra llave" y el lexema que expresa el sentido o el papel sintáctico de la FL, "valor de la FL"36.

Una vez expuesto someramente el instrumental teórico del que dispone el $D E C$ para describir las colocaciones, pasamos a una reflexión crítica del tratamiento de este fenómeno léxico, desde esta nueva perspectiva.

Frente al contextualismo británico, en este marco teórico, la frecuencia no tienen ninguna validez para considerar una determinada combinación como colocación. El hecho de que, por ejemplo, el lexema actividad se combine frecuentemente con febril es una consecuencia de que estos dos lexemas forman una colocación, no la causa.

La colocación es un fenómeno, en muchos casos, determinado léxicamente. De hecho, puede haber dos lexemas (cuasi-)sinónimos que tengan distintas relaciones colocacionales. Por ejemplo, compárese ganas incontenibles con deseo irresistible.

Los tipos de colocaciones léxicas distinguidas en el BBI son descritos también en el $D E C$ por FFLL. Con ellas se puede describir formal y semánticamente cada una de esas relaciones. Podemos mostrar aquí las correspondencias entre las colocaciones léxicas del $B B I$ y las FFLL del $D E C^{37}$ :

- tipo 1: Oper $_{1}$ (wound $)=$ inflict; Real $_{1}($ suspicion $)=$ confirm;

- tipo 2: Liqu $_{1}$ Func $_{2}($ license $)=$ revoke

- tipo 3: Magn(tea) = strong; Bon(regards) = warmest;

- tipo 4: Fact $_{0}$ (adjectives) = modify

- tipo 5: Mult $($ bee $)=$ colony; Sing $($ advice $)=a$ bit of

- tipo 6: Magn(absorbed $)=$ deeply

- tipo 7: $\operatorname{Magn}($ affect $)=$ deeply

Las colocaciones gramaticales del $B B I$ serían tratadas dentro del esquema de régimen. Ahí, se presentarán la realización superficial de los actantes del lexema descrito y las restricciones de coocurrencia de estos actantes. En el $D E C$, se delimita claramente la coocurrencia sintáctica de la léxica ya que obedecen a distintos criterios.

Benson et al..$^{38}$ critican las FFLL del $D E C$ por producir combinaciones libres. Como ejemplo, señalan que la FL Caus que proporciona el verbo to cause puede combinarse libremente con cientos de nombres. Ya hemos comentado este problema más arriba (vid. p. 20). Es cierto que en el $D E C$ han optado por incluir valores de una FL, que son

36 Obsérvese que la "base" y el "colocativo" de Hausmann se corresponden perfectamente con la palabra llave y el valor de una FL.

37 Los subíndices numéricos de algunas FLs aluden a los actantes de la palabra llave. Así, la FL Oper $_{1}$ (beso) $=$ dar, se leerá así: el actante I de beso (el que realiza la acción) será sujeto gramatical del verbo dar. Por otra parte, es probable que algunas de las colocaciones del $B B I$ fueran descritas por combinaciones de FFLL pero no entraremos aquí en eso.

38 Vid. BENSON et al. (1986b), p. 256. 
predecibles, con el fin de evitar que su ausencia fuera interpretada como inaceptabilidad de una colocación dada. Con todo, Caus no produce únicamente to cause sino también to establish, to seize, to place, to catch, to spark, to raise, to arouse, to inflict, etc. Por otra parte, to cause no se combina siempre libremente con un nombre dado: ? to cause joy / to arouse joy.

Otras dos FFLL son puestas en tela de juicio por estos autores. La FL Magn es criticada por ser demasiado productiva. Benson et al. ${ }^{39}$ piensan que no es deseable incluir en un diccionario, por ejemplo, la larga serie de adjetivos que expresan el sentido 'intensidad' predicado del nombre crime, en inglés. Nosotros pensamos que si un diccionario es exhaustivo, debe dar todos los valores de una FL aplicada a una palabra llave. Poco importa si el conjunto de valores es numeroso o no ${ }^{40}$.

Sobre la FL Func, Benson et al. (1986b) dicen que una aplicación indiscriminada de esta FL llevaría a combinaciones predecibles como bakers bake, boxers box. Frente a esto, debemos decir que estas combinaciones no serían proporcionadas por Func. Serían descritas por una FL paradigmática, $S_{1}$, que proporciona el nombre típico del primer actante. Así, en la entrada del verbo to bake, tendríamos la $\mathrm{FL} \mathrm{S}_{1}=$ baker.

Hasta cierto punto, las FLs corresponden al "lexis" de Halliday. Gran parte de ellas sirven para describir formalmente las colocaciones léxicas. Esta información es almacenada en el $D E C$, en el léxico teórico. Ahora bien, esa información es necesitada en todo el modelo lingüístico. Las FLs son operativas en el nivel de representación sintáctica para lexicalizar ciertos sentidos cuya expresión es arbitraria. Podríamos decir que las FFLL atraviesan la gramática. Asimismo, encontramos información gramatical en el propio sistema de las FFLL: basta con pensar en los índices actanciales, que ligan los actantes sintácticos profundos de la palabra llave con el valor de la FL.

El último aspecto que queremos tratar ahora con respecto a la exposición precedente es la composicionalidad semántica de las colocaciones. Por ejemplo, Cruse $\mathrm{e}^{41} \mathrm{y}$ Cumming ${ }^{42}$ mencionaban que, en las colocaciones, cada constituyente léxico es también un constituyente semántico. Pensamos que esto es cierto pero con algunas matizaciones. En una colocación, el elemento base guarda su sentido pero el colocativo tiene un sentido especial; este sentido sólo lo tiene en compañía de la base. Por ejemplo, vivo significa, aproximadamente, 'muy, en alto grado' cuando acompaña a interés pero, evidentemente, no cuando acompaña a hombre. Por tanto, sería más apropiado decir que las colocaciones son parcialmente composicionales. Frente a ellas, los frasemas son no composicionales y las combinaciones libres, completamente composicionales ${ }^{43}$.

39 Vid. Benson et al. (1986b), p. 257.

40 Es cierto que en el $D E C$ no se ha trazado una línea entre valores de una FL deducibles de otras informaciones (p. ej. sentir alegría) y valores que son completamente arbitrarios (p. ej. dar un paseo).

41 Vid. D. A. CRUSE (1986), p. 40.

42 Vid. S. Cumming (1986), p. 19.

43 Vid. U. HeId (1992), p. 219. 
Mel'cuk (1995a y 1995b) ${ }^{44}$ ha expuesto formalmente la diferencia entre esos tres tipos de combinaciones. Presentamos aquí su concepción, con algunas modificaciones.

Una combinación libre $A B$ es un sintagma compuesto de al menos dos lexemas A y $B$, de tal forma que su significado es la suma regular de los significados de los lexemas constituyentes y su significante es la suma regular de sus significantes.

La representación formal de una combinación libre sería la siguiente ${ }^{45}$ :

1)

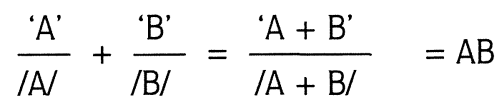

En prosa, la fórmula (1) significa que la unión del signo A, que tiene el significado ' $A$ ' y el significante $/ A$, con el signo $B$, que tiene el significado ' $B$ ' y el significante $/ B /$ produce una combinación libre $A B$, que tiene el significado ' $A+B$ ' y el significante $/ A+B /$.

En una combinación libre, el significante y el significado son construidos según las reglas generales de la lengua. Por ejemplo: comprar un coche, casa pequeña, etc.

Un frasema $A B$ es una combinación de al menos dos lexemas $A$ y $B$, de tal forma que sólo su significante es la suma regular de los significantes de los lexemas constituyentes. Su significado es diferente de la suma de los significados de los lexemas constituyentes; no incluye ni ' $A$ ' ni ' $B$ ' en una posición dominante.

La representación formal de un frasema es:

2)

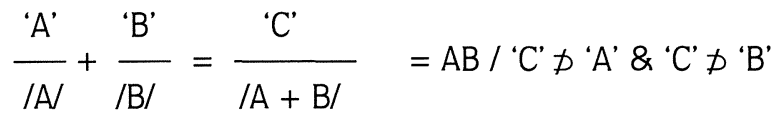

Como ejemplos de frasemas podemos ofrecer estirar la pata, tomar el pelo, con pelos y señales, ser el brazo derecho, etc.

Una colocación o "semi-frasema" (FL) AB es una combinación de dos lexemas A y $B$, de tal forma que su significante es la suma regular de los significantes de los lexemas constituyentes y su significado incluye el significado del lexema A y un significado 'C' que es:

44 En el $D E C$, el concepto de frasema no ha sido definido rigurosamente, aunque nunca se ha confundido con el concepto de colocación. Recientemente, Mel'čuk ha profundizado sobre la fraseología y ha establecido definiciones formales de todos los tipos de frasemas. En los dos trabajos mencionados, opta por utilizar la etiqueta "frasema" para todo sintagma no libre. El frasema incluye lo que en inglés se denomina "idioms" ( y que hasta ahora en el DEC y también nosotros en el texto, denominamos frasema), la colocación y los "cuasi-frasemas", de los que no hablaremos aquí. En los dos trabajos, Mel'čuk no presenta exactamente las mismas definiciones de estos conceptos. Nosotros las refundiremos pero sin entrar en todos los detalles.

45 Mel'ČuK (1995a) indica que en el uso de este tipo de fórmulas se ha inspirado en WEINRICH (1969), pp. 29 y ss. 
a) Bien 'C' 'B' y

- 'C' es vacío: el lexema B es un auxiliar usado para sostener una configuración sintáctica (dar un paseo);

- 'C' no es vacío pero el lexema B expresa 'C' sólo en combinación con 'A' o con otros pocos lexemas similares (odio mortal, interés vivo);

b) Bien ' $C$ ' = 'B' y

- El lexema $B$ es seleccionado restringidamente: en combinación con A no puede ser reemplazado por otro posible sinónimo (café fuerte <*potente>);

- 'C' incluye el sentido 'A' (pelo rubio, vino seco, nariz aguileña).

La representación formal de una colocación sería:

3)

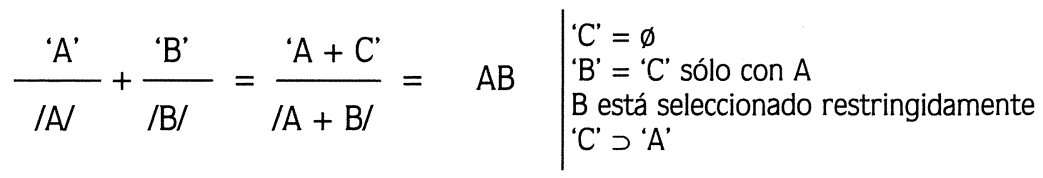

El lexema que guarda el sentido intacto será la palabra llave de la FL o la base de la colocación. Ejemplos de colocaciones serían interés vivo, ruido infernal, sueño ligero, nariz griega, vino dulce, dormir como un tronco, dar un paseo, cometer un crimen, etc.

Esta definición de colocación presenta las siguientes ventajas:

- Su formalización la hace más fácilmente operativa ya que facilita la descripción por medio del aparato de las FFLL: dado un lema, puede aplicársele la plantilla de las FLs sintagmáticas para describir todas las colocaciones en que ese lema aparezca como base o como palabra llave.

- No se apoya en criterios estadísticos sino puramente léxico-semánticos.

- Refleja la direccionalidad de las colocaciones en donde hay un lexema seleccionador y otro seleccionado.

- Marca claramente la delimitación con los frasemas o expresiones idiomáticas.

\section{BIBILIOGRAFÍA}

Alonso Ramos, M. (1989): “Aproximación a un nuevo modelo lexicográfico: El Dictionnaire explicatif et combinatoire du français contemporain. Recherches lexico-sémantiques de Igor Mel'čuk", Verba, 16, pp. 421-450.

Bally, CH. (1951): Traité de stylistique française, Paris, Klincksieck.

Bazell, C. E., J. C. Catford, M. A. K. Halliday y R. H. Robins(1966): In Memory of J. R. Firth, London, Longmann.

Béjolnt, H. y PH. Thoiron (1987), Compte rendu de Benson et al. (1986a): Les langues modernes, 81/3-4, pp. 152-159. 
Benson, M., E. Benson y R. ILSON (1986a): The BBI Combinatory Dictionary of English. A Guide to Word Combinations, Amsterdam-Philadelphia, John Benjamins.

Benson, M., E. BENSON y R. ILSON (1986b): The Lexicographic Description of English, Amsterdam, John Benjamins.

Coseriu, E. (1977): Principios de semántica estructural, Madrid, Gredos.

Cruse, D. A. (1986): Lexical Semantics, Cambridge, Cambridge University Press.

Cumming, S. (1986), “The Lexicon in Text Generation”, ISI Research Report ISI/RR-86-168, Information Sciences Institute, University of Southern California.

FIRTH, F. R. (1957): "Modes of Meaning”, en Papers in Linguistics 1934-1951, London, Oxford University Press, pp. 190-215.

Hausmann, F. (1979): “Un dictionnaie des collocations est-il possible?", Travaux de littérature et de linguistique de I'Université de Strasbourg, 17/1, pp. 187-195.

HallidaY, M. A. K. (1966): "Lexis as Linguistic Level", in BAZElL et al. (1966), pp. 148-162.

HallidAY, M. A. K. y R. HASAN (1976): Cohesion in English, London, Longmann.

HEID, U. (1992): "Notes on the Use of Lexical Functions for the Description of Collocations in an NLP Lexicon", in K.HAENELT y L. WANNER, eds., (1992): International Workshop on the Meaning-Text Theory, Schloss Birlinghoven, G.M.D., pp. 217-229.

HeID, U. y S. RAAB (1989): "Collocations in Multilingual Generation" en Proceedings of the 4th Meeting of the Association of Computational Linguistics, European Chapter.

Lyons, J. (1966): “Firth's Theory of 'Meaning'”, en BAzell et al. (1966), pp. 288-302.

Lyons, J. (1980): Semántica, Barcelona, Teide.

MeL'ČUK, I. A. (1995a): "Phrasemes in Language and Phraseology in Linguistics", en M. Everaert y A. Schenk, eds, Idioms, Erlbaum Associates.

Mel'čuk, I. A. (1995b): “The Future of the Lexicon in Linguistic Description: The Explanatory Combinatorial Dictionary", en I.-H. LeE, ed.,: Linguistics in the Morning Calm 3, Seoul.

Mel'čuk, I. A., N. ARBATChewSKY-Jumarie, L. ElnitSky, L. IORdansKaja y A. LeSSARd (1984): Dictionnaire explicatif et combinatoire du français contemporain. Recherches lexico-sémantiques I, Montréal, Les Presses de l'Université de Montreál.

Mel'čuk, I. A., N. ARBATCheWSKY-JumaRIE, L. DaGenais, L. Elnitsky, L. IoRdansKaja, M. N. LefeBVRE, y S. MANTHA (1988): Dictionnaire explicatif et combinatoire du français contemporain. Recherches lexico-sémantiques II, Montréal, Les Presses de l'Université de Montreál.

MeL'Čuk, I. A., N. ARBATCheWsky-Jumarie, L. IoRdanSKaJA y S. MANTHA (1992): Dictionnaire explicatif et combinatoire du français contemporain. Recherches lexico-sémantiques III, Montréal, Les Presses de l'Université de Montreál.

MeL'ČuK, I. A. y A. K. ŽolKovsKIJ (1970): “Towards a Functioning Meaning-Text Model of Language", Linguistics, 57, pp. 10-47.

MitchelL, T. F. (1971): “Linguistic 'Goings-On': Collocations and Other Lexical Matters Arising on the Syntagmatic Record", Archivum Linguisticum, 2, pp. 35-69.

Moliner, M. (1966-1967): Diccionario de uso del español, Madrid, Gredos. 
NiRENBURG, S. e I. NiRENBURG (1988): “A Framework for Lexical Selection in Natural Language Generation", Proceedings of COLING-88, Budapest, pp. 471-475.

RoBINs, R. H. (1971): Lingüística general, Gredos, Madrid.

Salvador, G. (1989-90): "Las solidaridades lexemáticas", Revista de Filología de la Universidad de La Laguna, pp. 339-365.

WeInREICH, U. (1969): "Problems in the analysis of idioms", en J. Punvel, ed., Substance and Structure of Language, Berkeley-Los Angeles, University of California Press, pp. 23-81. 